\title{
AN ENERGY GAP FOR YANG-MILLS CONNECTIONS
}

\author{
CLAUS GERHARDT
}

\begin{abstract}
Consider a Yang-Mills connection over a Riemann manifold $M=M^{n}, n \geq 3$, where $M$ may be compact or complete. Then its energy must be bounded from below by some positive constant, if $M$ satisfies certain conditions, unless the connection is flat.
\end{abstract}

\section{Contents}

1. Introduction

2. The compact case

3. The non-compact case

References

\section{INTRODUCTION}

We consider the problem: When is a Yang-Mills connection non-flat? Of course, the trivial answer $F_{\mu \lambda} \not \equiv 0$ is unsatisfactory. Bourguignon and Lawson proved in [3, Theorem $\mathrm{C}$ ], among other results, that any Yang-Mills connection over $S^{n}, n \geq 3$, the field strength of which satisfies the pointwise estimate

$$
F^{2}=-\operatorname{tr}\left(F_{\mu \lambda} F^{\mu \lambda}\right)<\left(\begin{array}{l}
n \\
2
\end{array}\right)
$$

is flat.

We want to prove that under certain assumptions on the base space $M$, which is supposed to be a Riemannian manifold of dimension $n \geq 3$, the energy of a Yang-Mills connection has to satisfy

$$
\left(\int_{M}|F|^{\frac{n}{2}}\right)^{\frac{2}{n}} \geq \kappa_{0}>0,
$$

where $\kappa_{0}$ depends only on the Sobolev constants of $M, n$ and the dimension of the Lie group $\mathcal{G}$, unless the connection is flat.

Date: July 18, 2018.

2000 Mathematics Subject Classification. 35J60, 53C21, 53C44, 53C50, 58J05.

Key words and phrases. energy gap, Yang-Mills connections.

This work has been supported by the DFG. 
Here,

$$
|F|=\sqrt{F^{2}},
$$

and we also call the left-hand side of (1.2) energy though this label is only correct when $n=4$. However, this norm is also the crucial norm, which has to be (locally) small, used to prove regularity of a connection, cf. 4, Theorem 1.3 .

The exponent $\frac{n}{2}$ naturally pops up when Sobolev inequalities are applied to solutions of differential equations satisfied by the field strength or the energy density of a connection in the adjoint bundle.

We distinguish two cases: $M$ compact and $M$ complete and non-compact. When $M$ is compact, we require

$$
\bar{R}_{\alpha \beta} \Lambda_{\lambda}^{\alpha} \Lambda^{\beta \lambda}-\frac{1}{2} \bar{R}_{\alpha \beta \mu \lambda} \Lambda^{\alpha \beta} \Lambda^{\mu \lambda} \geq c_{0} \Lambda_{\alpha \beta} \Lambda^{\alpha \beta}
$$

for all skew-symmetric $\Lambda_{\alpha \beta} \in T^{0,2}(M)$, where $0<c_{0}$, while for non-compact $M$ the weaker assumption

$$
\bar{R}_{\alpha \beta} \Lambda_{\lambda}^{\alpha} \Lambda^{\beta \lambda}-\frac{1}{2} \bar{R}_{\alpha \beta \mu \lambda} \Lambda^{\alpha \beta} \Lambda^{\mu \lambda} \geq 0
$$

and in addition

$$
\left(\int_{M} u^{\frac{2 n}{n-2}}\right)^{\frac{n-2}{n}} \leq c_{1} \int_{M}|D u|^{2} \quad \forall u \in H^{1,2}(M)
$$

should be satisfied.

1.1. Remark. (i) If $M$ is a space of constant curvature

$$
\bar{R}_{\alpha \beta \mu \lambda}=K_{M}\left(\bar{g}_{\alpha \mu} \bar{g}_{\beta \lambda}-\bar{g}_{\alpha \lambda} \bar{g}_{\beta \mu}\right),
$$

then

$$
\bar{R}_{\alpha \beta} \Lambda_{\lambda}^{\alpha} \Lambda^{\beta \lambda}-\frac{1}{2} \bar{R}_{\alpha \beta \mu \lambda} \Lambda^{\alpha \beta} \Lambda^{\mu \lambda}=(n-2) K_{M} \Lambda_{\alpha \beta} \Lambda^{\alpha \beta} .
$$

In case $n=2$ the curvature term therefore vanishes, and this result is also valid for an arbitrary two-dimensional Riemannian manifold, since the curvature tensor then has the same structure as in (1.7) though $K_{M}$ is not necessarily constant.

(ii) If $M=\mathbb{R}^{n}, n \geq 3$, the conditions (1.5) and (1.6) are always valid.

1.2. Theorem. Let $M=M^{n}, n \geq 3$, be a compact Riemannian for which the condition (1.4) with $c_{0}>0$ holds. Then any Yang-Mills connection over $M$ with compact, semi-simple Lie group is either flat or satisfies (1.2) for some constant $\kappa_{0}>0$ depending on the Sobolev constants of $M, n, c_{0}$, and the dimension of the Lie group.

1.3. Theorem. Let $M=M^{n}, n \geq 3$, be complete, non-compact and assume that the conditions (1.5) and (1.6) hold. Then any Yang-Mills connection over $M$ with compact, semi-simple Lie group is either flat or the estimate (1.2) is valid. The constant $\kappa_{0}>0$ in (1.2) depends on the constant $c_{1}$ in (1.6), $n$, and the dimension of the Lie group. 


\section{The COMPACT CASE}

Let $(P, M, \mathcal{G}, \mathcal{G})$ be a principal fiber bundle where $M=M^{n}, n \geq 3$ is a compact Riemannian manifold with metric $\bar{g}_{\alpha \beta}$ and $\mathcal{G}$ a compact, semi-simple Lie group with Lie algebra $\mathfrak{g}$. Let $f_{c}=\left(f_{c b}^{a}\right)$ be a basis of ad $\mathfrak{g}$ and

$$
A_{\mu}=f_{c} A_{\mu}^{c}
$$

a Yang-Mills connection in the adjoint bundle $(E, M, \mathfrak{g}, \operatorname{Ad}(\mathcal{G}))$.

The curvature tensor of the connection is given by

$$
R_{b \mu \lambda}^{a}=f_{c b}^{a} F_{\mu \lambda}^{c},
$$

where

$$
F_{\mu \lambda}=f_{c} F_{\mu \lambda}^{c}
$$

is the field strength of the connection, and

$$
F^{2} \equiv \gamma_{a b} F_{\mu \lambda}^{a} F^{b \mu \lambda}=R_{a b \mu \lambda} R^{a b \mu \lambda}
$$

the energy density of the connection - at least up to a factor $\frac{1}{4}$.

Here, $\gamma_{a b}$ is the Cartan-Killing metric acting on elements of the fiber $\mathfrak{g}$, and Latin indices are raised or lowered with respect to the inverse $\gamma^{a b}$ or $\gamma_{a b}$, and Greek indices with respect to the metric of $M$.

2.1. Definition. The adjoint bundle $E$ is vector bundle; let $E^{*}$ be the dual bundle, then we denote by

$$
T^{r, s}(E)=\Gamma(\underbrace{E \otimes \cdots \otimes E}_{r} \otimes \underbrace{E^{*} \otimes \cdots \otimes E^{*}}_{s})
$$

the sections of the corresponding tensor bundle.

Thus, we have

$$
F_{\mu \lambda}^{a} \in T^{1,0}(E) \otimes T^{0,2}(M) .
$$

Since $A_{\mu}$ is a Yang-Mills connection it solves the Yang-Mills equation

$$
F_{\lambda ; \alpha}^{a \alpha}=0,
$$

where we use Einstein's summation convention, a semi-colon indicates covariant differentiation, and where we stipulate that a covariant derivative is always a full tensor, i.e.,

$$
F_{\mu \lambda ; \alpha}^{a}=F_{\mu \lambda, \alpha}^{a}+f_{b c}^{a} A_{\alpha}^{b} F_{\mu \lambda}^{c}-\bar{\Gamma}_{\alpha \mu}^{\gamma} F_{\gamma \lambda}^{a}-\bar{\Gamma}_{\alpha \lambda}^{\gamma} F_{\mu \gamma}^{a},
$$

where $\bar{\Gamma}_{\alpha \beta}^{\gamma}$ are the Christoffel symbols of the Riemannian connection; a comma indicates partial differentiation.

Before we formulate the crucial lemma let us note that $\bar{R}_{\alpha \beta \gamma \delta}$ resp. $\bar{R}_{\alpha \beta}$ symbolize the Riemann curvature tensor resp. the Ricci tensor of $\bar{g}_{\alpha \beta}$. 
2.2. Lemma. Let $A_{\mu}$ be a Yang-Mills connection, then its energy density $F^{2}$ solves the equation

$$
\begin{aligned}
& -\frac{1}{4} \Delta F^{2}+\frac{1}{2} F_{a \mu \lambda ; \alpha} F^{a \mu \lambda \alpha}+\bar{R}_{\beta \mu} F_{\lambda}^{a \beta} F_{a}{ }^{\mu \lambda}-\frac{1}{2} \bar{R}_{\alpha \beta \mu \lambda} F_{a}{ }^{\alpha \beta} F^{a \mu \lambda} \\
& =-f_{c b}^{a} F_{\alpha \mu}^{c} F_{\lambda}^{b \alpha} F_{a}{ }^{\mu \lambda} .
\end{aligned}
$$

Proof. Differentiating (2.7) covariantly with respect to $x^{\mu}$ and using the Ricci identities we obtain

$$
\begin{aligned}
0 & =-F_{\lambda ; \alpha \mu}^{a \alpha} \\
& =-F_{\lambda ; \mu \alpha}^{a \alpha}+R_{b \alpha \mu}^{a} F_{\lambda}^{b \alpha}+\bar{R}_{\beta \alpha \mu}^{\alpha} F_{\lambda}^{a \beta}+\bar{R}_{\lambda \mu \alpha}^{\beta} F_{\beta}^{a \alpha} .
\end{aligned}
$$

On the other hand, differentiating the second Bianchi identities

$$
0=F_{\alpha \lambda ; \mu}^{a}+F_{\mu \alpha ; \lambda}^{a}+F_{\lambda \mu ; \alpha}^{a}
$$

we infer

$$
0=F_{\lambda ; \mu \alpha}^{a \alpha}+F_{\mu ; \lambda \alpha}^{a}{ }^{\alpha}+\Delta F_{\lambda \mu}^{a},
$$

and we deduce further

$$
-\Delta F_{\mu \lambda}^{a} F_{a}{ }^{\mu \lambda}=-2 F_{\lambda ; \mu \alpha}^{a \alpha} F_{a}{ }^{\mu \lambda} .
$$

In view of (2.10) we then conclude

$$
\begin{aligned}
0=-\frac{1}{2} \Delta F_{\mu \lambda}^{a} F_{a}{ }^{\mu \lambda} & +R_{b \alpha \mu}^{a} F_{\lambda}^{b \alpha} F_{a}{ }^{\mu \lambda}+\bar{R}_{\beta \mu} F_{\lambda}^{a \beta} F_{a}{ }^{\mu \lambda} \\
& +\bar{R}_{\lambda \mu \alpha}^{\beta} F_{\beta}^{a \alpha} F_{a}{ }^{\mu \lambda},
\end{aligned}
$$

which is equivalent to

$$
\begin{aligned}
0=-\frac{1}{2} \Delta F_{\mu \lambda}^{a} F_{a}{ }^{\mu \lambda} & +f_{c b}^{a} F_{\alpha \mu}^{c} F_{\lambda}^{b \alpha} F_{a}{ }^{\mu \lambda}+\bar{R}_{\beta \mu} F_{\lambda}^{a \beta} F_{a}{ }^{\mu \lambda} \\
& -\bar{R}_{\alpha \mu \beta \lambda} F^{a \alpha \beta} F_{a}{ }^{\mu \lambda},
\end{aligned}
$$

in view of (2.2).

Finally, using the first Bianchi identities,

$$
\bar{R}_{\alpha \beta \mu \lambda}+\bar{R}_{\alpha \mu \lambda \beta}+\bar{R}_{\alpha \lambda \beta \mu}=0,
$$

we deduce

$$
\bar{R}_{\alpha \beta \mu \lambda} F^{a \alpha \beta} F_{a}{ }^{\mu \lambda}+\bar{R}_{\alpha \mu \lambda \beta} F^{a \alpha \beta} F_{a}{ }^{\mu \lambda}+\bar{R}_{\alpha \lambda \beta \mu} F^{a \alpha \beta} F_{a}{ }^{\mu \lambda}=0,
$$

and hence

$$
\bar{R}_{\alpha \beta \mu \lambda} F^{a \alpha \beta} F_{a}{ }^{\mu \lambda}=2 \bar{R}_{\alpha \mu \beta \lambda} F^{a \alpha \beta} F_{a}{ }^{\mu \lambda},
$$

from which the equation (2.9) immediately follows.

Proof of Theorem 1.2 on page 2. Define

$$
u=F^{2} \text {, }
$$

then

$$
\bar{R}_{\beta \mu} F_{\lambda}^{a \beta} F_{a}{ }^{\mu \lambda}-\frac{1}{2} \bar{R}_{\alpha \beta \mu \lambda} F_{a}{ }^{\alpha \beta} F^{a \mu \lambda} \geq c_{0} u,
$$

where $c_{0}>0$, in view of the assumption (1.4) on page 2 
Multiplying (2.9) with $u$ and integrating by part we obtain

$$
\frac{3}{8} \int_{M}|D u|^{2}+c_{0} \int_{M} u^{2} \leq c \int_{M} \sqrt{u} u^{2},
$$

where we used the simple estimate

$$
|D u|^{2} \leq 4 F_{a \mu \lambda ; \alpha} F_{;}^{a \mu \lambda}{ }^{\alpha} u^{2}
$$

and where $c$ depends on $n$ and the dimension of $\mathfrak{g}$; note that

$$
f_{c} \in \operatorname{SO}\left(\mathfrak{g}, \gamma_{a b}\right) \text {. }
$$

The integral on the right-hand side of (2.21) is estimated by

$$
\int_{M} \sqrt{u} u^{2} \leq\left(\int_{M} u^{\frac{n}{4}}\right)^{\frac{2}{n}}\left(\int_{M} u^{\frac{2 n}{n-2}}\right)^{\frac{n-2}{n}},
$$

where

$$
\left(\int_{M} u^{\frac{n}{4}}\right)^{\frac{2}{n}}=\left(\int_{M}|F|^{\frac{n}{2}}\right)^{\frac{2}{n}} .
$$

Applying then the Sobolev inequality

$$
\left(\int_{M} u^{\frac{2 n}{n-2}}\right)^{\frac{n-2}{n}} \leq c_{1} \int_{M}|D u|^{2}+c_{2} \int_{M} u^{2},
$$

cf. [1, we obtain

$$
\left(\int_{M} u^{\frac{2 n}{n-2}}\right)^{\frac{n-2}{n}} \leq c_{3}\left(\int_{M}|F|^{\frac{n}{2}}\right)^{\frac{2}{n}}\left(\int_{M} u^{\frac{2 n}{n-2}}\right)^{\frac{n-2}{n}},
$$

where $c_{3}$ depends on $c_{1}, c_{2}, c_{0}$ and $c$. Hence, we deduce $u \equiv 0$ or

$$
c_{3}^{-1} \leq\left(\int_{M}|F|^{\frac{n}{2}}\right)^{\frac{2}{n}} .
$$

Setting

$$
\kappa_{0}=c_{3}^{-1}
$$

finishes the proof.

\section{THE NON-COMPACT CASE}

We now suppose that $M=M^{n}$ is a complete, non-compact Riemannian manifold. Then there holds

$$
H^{1,2}(M)=H_{0}^{1,2}(M),
$$

i.e., the test functions $C_{c}^{\infty}(M)$ are dense in the Sobolev space $H^{1,2}(M)$, see [1, Lemme 4] or [2, Theorem 2.6].

Since we do not a priori

$$
F^{2} \in H^{1,2}(M)
$$

but only

$$
F^{2} \in H_{\mathrm{loc}}^{1,2}(M),
$$


the preceding proof has to be modified.

Let $\eta=\eta(t)$ be defined through

$$
\eta(t)= \begin{cases}1, & t \leq 1 \\ (2-t)^{q}, & 1 \leq t \leq 2 \\ 0, & t \geq 2\end{cases}
$$

where

$$
q=\max \left(1, \frac{8}{n}\right) .
$$

Fix a point $x_{0} \in M$ and let $r$ be the Riemannian distance function with center in $x_{0}$

$$
r(x)=d\left(x_{0}, x\right) .
$$

Then $r$ is Lipschitz such that

$$
|D r|=1
$$

almost everywhere.

For $k \geq 1$ define

$$
\eta_{k}(x)=\eta\left(k^{-1} r\right) .
$$

The functions

$$
u^{p-1} \eta_{k}^{p}
$$

where

$$
p=\frac{n}{4},
$$

then have compact support, and multiplying (2.9) on page 4 with $u^{p-1} \eta_{k}^{p}$ yields

$$
\begin{aligned}
\left(\frac{p}{4}+\frac{1}{8}-\epsilon\right) \int_{m}|D u|^{2} u^{p-2} \eta_{k}^{p} \leq c & \left(\int_{M}|F|^{\frac{n}{2}}\right)^{\frac{2}{n}}\left(\int_{M}\left(u \eta_{k}\right)^{\frac{n}{n-2} p}\right)^{\frac{n-2}{n}} \\
& +c_{\epsilon} \int_{M}\left|D \eta_{k}\right|^{2} \eta_{k}^{p-2} u^{p}
\end{aligned}
$$

where $0<\epsilon$ is supposed to be small.

Furthermore, there holds

$$
\begin{aligned}
\int_{M}\left|D\left(u \eta_{k}\right)^{\frac{p}{2}}\right|^{2} & =\frac{p^{2}}{4} \int_{M}\left|D u \eta_{k}+u D \eta_{k}\right|^{2}\left(u \eta_{k}\right)^{p-2} \\
\leq & (1+\epsilon) \frac{p^{2}}{4} \int_{M}|D u|^{2} u^{p-2} \eta_{k}^{p}+c_{\epsilon} \frac{p^{2}}{4} \int_{M}\left|D \eta_{k}\right|^{2} \eta_{k}^{p-2} u^{p} .
\end{aligned}
$$

Now, choosing $\epsilon$ so small that

$$
(1+\epsilon) \frac{p^{2}}{4} \leq p\left(\frac{p}{4}+\frac{1}{8}-\epsilon\right)
$$

and setting

$$
\varphi=\left(u \eta_{k}\right)^{\frac{p}{2}}
$$


we obtain

$$
\int_{M}|D \varphi|^{2} \leq p c\left(\int_{M}|F|^{\frac{n}{2}}\right)^{\frac{2}{n}}\left(\int_{M} \varphi^{\frac{2 n}{n-2}}\right)^{\frac{n-2}{n}}+c_{\epsilon} \int_{M}\left|D \eta_{k}\right|^{2} \eta_{k}^{p-2} u^{p},
$$

where $c_{\epsilon}$ is a new constant.

We furthermore observe that

$$
\left|D \eta_{k}\right|^{2} \eta_{k}^{p-2} \leq q^{2} k^{-2}\left(2-k^{-1} r\right)^{q p-2},
$$

subject to

$$
1 \leq k^{-1} r \leq 2
$$

In view of (3.5) and (3.10)

$$
q p-2 \geq 0
$$

and hence

$$
\left|D \eta_{k}\right|^{2} \eta_{k}^{p-2} \leq q^{2} k^{-2}
$$

Applying now the Sobolev inequality (1.6) on page 2 to $\varphi$ and choosing

$$
\kappa_{0}=\left(c_{1} c p\right)^{-1}
$$

we conclude $|F| \equiv 0$, if

$$
\left(\int_{M}|F|^{\frac{n}{2}}\right)^{\frac{2}{n}}<\kappa_{0}
$$

Indeed, if the preceding inequality is valid, then we deduce from (3.15)

$$
\left(1-\kappa_{0}^{-1}\left(\int_{M}|F|^{\frac{n}{2}}\right)^{\frac{2}{n}}\right)\left(\int_{M}|\varphi|^{\frac{2 n}{n-2}}\right)^{\frac{n-2}{n}} \leq c_{\epsilon} q^{2} k^{-2} \int_{M}|F|^{\frac{n}{2}} .
$$

In the limit $k \rightarrow \infty$ we obtain

$$
\left(\int_{M}|u|^{\frac{p n}{n-2}}\right)^{\frac{n-2}{n}} \leq 0
$$

\section{REFERENCES}

[1] Thierry Aubin, Problèmes isopérimétriques et espaces de Sobolev, J. Differential Geometry 11 (1976), no. 4, 573-598.

[2] Nonlinear analysis on manifolds. Monge-Ampère equations, Grundlehren der Mathematischen Wissenschaften [Fundamental Principles of Mathematical Sciences], vol. 252, Springer-Verlag, New York, 1982.

[3] Jean-Pierre Bourguignon and H. Blaine Lawson, Jr., Stability and isolation phenomena for Yang-Mills fields, Comm. Math. Phys. 79 (1981), no. 2, 189-230.

[4] Karen K. Uhlenbeck, Connections with $L^{p}$ bounds on curvature, Comm. Math. Phys. 83 (1982), no. 1, 31-42.

Ruprecht-Karls-Universität, Institut für Angewandte Mathematik, Im NeuenHeimer Feld 294, 69120 Heidelberg, Germany

E-mail address: gerhardt@math.uni-heidelberg.de

URL: http://www.math.uni-heidelberg.de/studinfo/gerhardt/ 